\title{
Clinical Response of Whole Blood Transfusion for the Treatment of Theileria Associated Bovine Anemia (TABA) in a Crossbred Jersey Cows
}

\section{S Yogeshpriya*, P Selvaraj, N Prmelatha, M Saravanan, M Veeraselvam and $\mathrm{K}$ Jayalakshmi}

Large Animal Medicine Referral Clinic, Veterinary College and Research Institute, Tamilnadu Veterinary and Animal Sciences University, India

*Corresponding Author: S Yogeshpriya, Large Animal Medicine Referral Clinic, Veterinary College and Research Institute, Tamilnadu Veterinary and Animal Sciences University, India.
Received: August 30, 2021

Published: October 08, 2021

(C) All rights are reserved by $\mathbf{S}$ Yogeshpriya., et al.

\begin{abstract}
In a study of 24 anemic cows, which were screened for hemoparasites during the year 2017, 10 were found to have Theileria sp. and among that 6 animals were found to have highly positive and those cows were also severe anemic. Owners failed to report for treatment in a timely manner, and all six cows finally acquired anaemia. Point of care hematology revealed reduced hemoglobin, Packed cell volume and total erythrocyte counts. A single dose of inj. Buparvaquone was given to all of the animals @ $2.5 \mathrm{mg} / \mathrm{kg}$ i/m on the day of presensentation along with oxytetracycline @10 mg/kg body weight. Studies in the field of hemotherapy for ruminants are scarce, particularly in relation to blood conservation and responses to transfusion. Based on the laboratory and clinical findings, subsequently all the 6 animals were managed with the aid of whole blood transfusion and other supportive treatment.
\end{abstract}

Keywords: Anemia; Theileria; Transfusion; TABA

Hemoparasitic illnesses transmitted by arthropods are economically significant vector-borne diseases in tropical and subtropical areas of the world. Bovine theileriosis is a tick-borne hemoprotozoan disease that affects cattle and is caused by a variety of theileria species. The pathogenic and commercially significant $T$. parva, which causes East Coast fever, and T. annulata, which causes tropical theileriosis, are the most pathogenic and economically important. The sporozoites are also spread to animals by the saliva of the feeding tick. High fever, weakness, weight loss, inappropriate hunger, conjunctival petechia, swollen lymph nodes, and anaemia are all symptoms of bovine theileriosis. In anaemic patients, blood transfusion is the most effective treatment.
Blood typing is not achievable in cattle due to the enormous amount of red cell antigens, therefore finding a perfect donor is nearly impossible. To protect against transfusion response, cross matching is used instead of blood typing. Due to the lack of naturally occurring alloantibodies, initial transfusions in cattle are generally regarded safe, even when done without cross matching (Smith, 2002). It is detrimental to the production economics due to extensive death losses, decreased milk and meat production, reproductive problems and increasing risk of secondary infections [1]. For these reasons, choosing the most effective, specific and supportive treatment for the disease gains major importance. 


\section{Materials and Methods}

The study was carried out in Large Animal Medicine Referral Clinic, Veterinary College and Research Institute, Orathanadu during the year 2017. The 24 animals suspected of having hemoparasites and having a history of respiratory distress, anorexia, and watery diarrhoea with foul odour were chosen for this study. On physical examination, six of the animals had icteric mucous membranes and enlargement of the prescapular lymph node. Lymph node aspirates were taken from swollen lymph nodes and utilised to do a lymph smear. Smears of blood and lymph nodes were made immediately after collection, fixed with $100 \%$ methanol, and stained with Giemsa. Before and after treatment, blood was collected in sterile tubes coated with the disodium salt of ethylenediaminetetraacetic acid as an anticoagulant. The blood samples were subjected for determination of hemoglobin ( $\mathrm{Hb})$, packed cell volume (PCV), total erythrocyte count (TEC), and total leukocyte count (TLC) of the cattle's suffering from theileriosis. For biochemical studies, blood was collected in clot activator vial thus sera were separated and kept immediately in the deep freezer at $-20^{\circ} \mathrm{C}$ till analysis and was used for estimation of various biochemical parameters like total protein, albumin (Al), globulin (G), glucose, aspartate aminotransferase (AST), and alanine aminotransferase (ALT), using commercially available kits. All statistical data were analyzed as per the standard methods.

Regardless of the donor, the first blood transfusion in cattle should usually be safe. A crossmatching blood compatibility test was performed between the donor and the recipient prior to blood transfusion. Fresh blood that had been collected in CPDA-bags was transfused. The blood bags were taken out of the refrigerator for the transfusion, and after 30 minutes at room temperature, the blood was transfused to the animals.

\section{Results and Discussion}

Out of 24 animals blood smears screened for hemoparasites, 6 animals harboured Theileria annulata organisms by Giemsa's technique. Disease is characterized by high fever (rectal temperature $>39^{\circ} \mathrm{C}$ ), enlargement of superficial lymph nodes, anaemia, jaundice, drop in milk production. In severe cases there is laboured breathing, with rapid and shallow breaths followed by increased heart rate. Microscopic examination of stained peripheral thin blood smears revealed Theileria annulata (Figure 1). All animals with enlarged lymph nodes were positive for theileria infection by lymph smear, infected lymphocyte cells showed schizonte stage of the parasite which called Koch's blue bodies as in figure 2. Haemoglobinurea was not observed in any of the cases under study. The destruction of piroplasm infected erythrocytes by macrophages in the lymph nodes, spleen, and other organs of the monocyte macrophage system, coupled with reduced erythropoietic activity, the toxic effects of Theileria species metabolites, persistent blood loss caused by permanent bloodsucking ticks, and TNF- on eosinophils are all possible explanations for the decline in $\mathrm{Hb}$, TEC, and PCV (Table 1).

Theileriosis animals had significantly higher AST and ALT levels than healthy animals. These findings back up Hasanpour., et al. [2] findings from their investigations on theileriosis. Parasitic tis-

\begin{tabular}{|l|c|c|c|c|c|c|}
\hline Variables & Day 0 & Day 2 (B.T) & Day3 (A.T) & Day 5(A.T) & Day 7(A.T) & Day 10(A.T) \\
\hline $\mathrm{Hb}(\mathrm{g} / \mathrm{dl})$ & $3 \pm 1.24^{*}$ & $6.2 \pm 0.94$ & $6.6 \pm 0.90$ & $7.2 \pm 0.12$ & $7.9 \pm 1.17$ & $9.5 \pm 1.11^{*}$ \\
\hline $\mathrm{PCV}(\%)$ & $12 \pm 0.18^{*}$ & $18 \pm 1.10$ & $21 \pm 0.98$ & $22 \pm 0.36$ & $24 \pm 0.45$ & $30.8 \pm 1.27^{*}$ \\
\hline $\mathrm{TEC}\left(\times 10^{6} / \mu \mathrm{l}\right)$ & $1.99 \pm 1.12^{*}$ & $4.12 \pm 0.32$ & $5.01 \pm 1.10$ & $5.2 \pm 0.63$ & $6.0 \pm 0.71$ & $6.9 \pm 0.59^{*}$ \\
\hline $\mathrm{TLC}\left(\times 10^{3} / \mu \mathrm{l}\right)$ & $3.59 \pm 0.8^{*}$ & $8 \pm 0.51$ & $8 \pm 0.77$ & $8.2 \pm 0.13$ & $8.2 \pm 1.10$ & $8.49 \pm 0.43^{*}$ \\
\hline
\end{tabular}

Table 1

*A.T- After Whole blood transfusion, *B.T- Before Whole blood transfusion.

sue damage is caused by the presence of parasites in any tissue. Increased serum activities of AST and ALT are closely connected with hepatic function in theileriosis, including total protein, albu- min (Al), globulin (G), glucose, aspartate aminotransferase (AST), and alanine aminotransferase (ALT) (ALT). 
The donor's choice may be limited by availability, but it should be a healthy, mature animal that can be handled. For all of the examples given, apparently healthy cross-bred jersey cattle were chosen, and a blood profile was performed to rule out infection, as well as heamato biochemical examination, which were both found to be within normal limits. There was no evidence of hemoprotozoan illness in any of the donors. Cattle have a total blood volume of 7-8 percent of their body weight. At any given time, no more than $25 \%$ of a donor's entire blood volume should be taken. For most indications, 10-15 percent of an adult's blood volume (5-6 litres) is usually sufficient. Throughout the transfusion, clinical observations were recorded, and heart rate, breathing rate, and rectal temperature were measured.

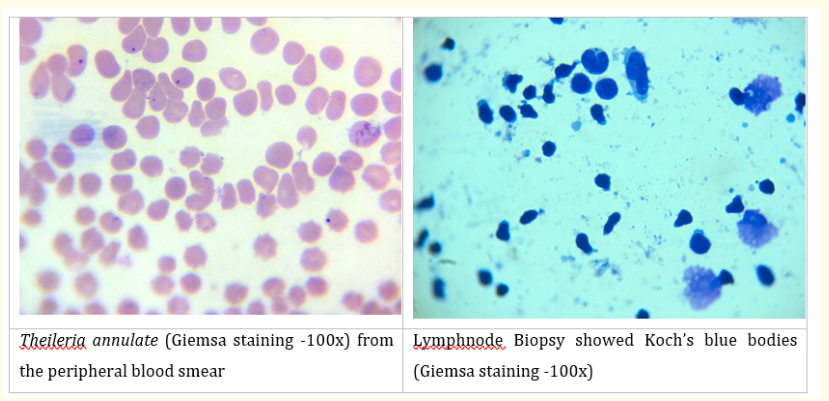

Figure 1

In this investigation, a single injection of Buparvaquone @ 2.5 $\mathrm{mg} / \mathrm{kg}$ b.wt. intramuscularly was used as an adjunct to buparvaquone, and oxytetracycline @ $10 \mathrm{mg} / \mathrm{kg}$ b.wt. intravenously was used daily for five days to cure respiratory infection in cattle. To treat anaemia, blood transfusions were planned for all six anaemic cows. A donor animal that appeared to be healthy was chosen and screened for haemoprotozoan illnesses. For the donated animal, a comprehensive haematological and serum examination was performed. The blood was tested for compatibility, and $1500 \mathrm{~mL}$ of blood was obtained and successfully transfused. The electrocardiogram was used to carefully monitor the blood transfusion as per the normal practise [3]. Hematology blood samples were taken on a regular basis and evaluated for changes in Erythron levels. Blood was obtained for haematological examination eight days following the blood transfusion, and the results were normal (Table 1).

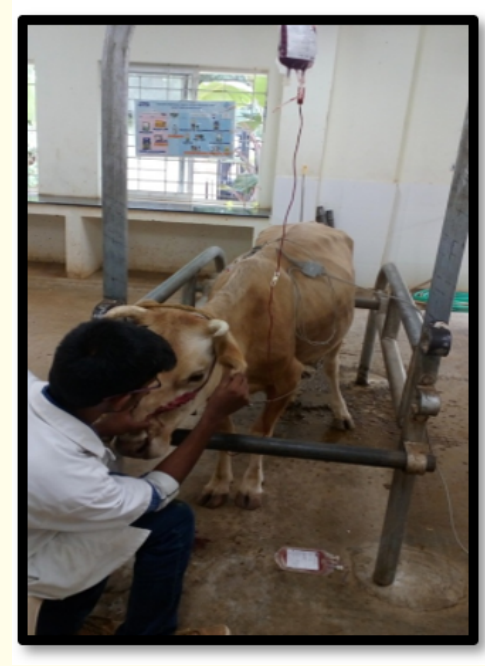

Figure 2: Whole blood transfusion under cardiac monitoring.

Supportive treatment with Vitamin B-complex with liver extract, haematinic, and fluid therapy can help theileriosis cattle recover more quickly and correct their negative energy balance.

Any unfavourable response after receiving entire blood or blood components is referred to as a post-transfusion reaction. Tachycardia, tachypnea, tremors, fever, pruritus, dyspnea, hematuria, and hemoglobinuria are among the most prevalent indications of transfusion responses in ruminants. However, because ruminants have low amounts of circulating isoantibodies, some publications indicate that transfusion responses in ruminants following a single blood transfusion are unusual. However, none of the cattle in this trial had any negative effects $[4,5]$.

\section{Conclusion}

In severe anaemia, transfusion treatment is an important element of veterinary emergency and critical care medicine. When severe anaemia occurs, the blood's oxygen carrying capacity is depleted to the point where life is jeopardised, blood transfusion is needed. The majority of the animals die from severe anaemia. Blood and blood products can be obtained by purchasing blood products or by donating blood. As a result, in cases of life-threatening anaemia in cattle, a simple, rapid, economical, and timely delivery of blood is clinically extremely rewarding. 


\section{Acknowledgement}

The authors are highly thankful to the Dean, Veterinary College and Research Institute, Orathanadu, TANUVAS, for providing the necessary inputs for successful conduct of research.

\section{Bibliography}

1. Radostits OM., et al. "Veterinary Medicine. A Textbook of Diseases of Cattle, Sheep, Goat and Horses". 10th edition, Newyork WB Saunders Company ltd. Smith, B. P. (2002). Large Animal Internal Medicine 3rd edn., Mosby St. Louis Missouri, U.S.A (2007).

2. Hasanpour A., et al. "Biochemical, Hematological, and Electrocardiographic Changes in Buffaloes Naturally Infected with Theileria annulate". Korean Journal of Parasitology 46.4 (2008): 223-227.

3. S Yogeshpriya., et al. "ECG Markers in Subclinical Hypocalcaemia Exacerbated Clinical Ketosis in Transition Cows". Indian Veterinary Journal 96.5 (2019): 47-49.

4. Saravanan M., et al. "Clinical, Hematological Changes and Therapeutic Efficacy of Buparvaquone with Oxytetracycline against the Natural Infection of Theileria Annulata in Cattle". International Journal of Livestock Research 7.10 (2017): 128-133.

5. Yogeshpriya S., et al. "Haemato- biochemical and electrolyte alterations in naturally occurring theileria associated bovine anaemia (taba)". Journal of Animal Health and Production 5.2 (2017): 64-67.

\section{Assets from publication with us}

- Prompt Acknowledgement after receiving the article

- Thorough Double blinded peer review

- Rapid Publication

- Issue of Publication Certificate

- High visibility of your Published work

Website: www.actascientific.com/

Submit Article: www.actascientific.com/submission.php

Email us: editor@actascientific.com

Contact us: +919182824667

Citation: S Yogeshpriya., et al. "Clinical Response of Whole Blood Transfusion for the Treatment of Theileria Associated Bovine Anemia (TABA) in a Crossbred Jersey Cows". Acta Scientific Veterinary Sciences 4.1 (2022): 11-14. 